fand sich zweimal. Am häufigsten (viermal) kam eine Verdoppelung des Sesamoids vor.

Vier Sesamoide wurden außerdem nur noch zweimal (bei demselben Individuum) an Doppelgroßzehen beobachtet, an welchen letzteren eine vollkommene Syndaktylie bestand; die beiden mittleren Knöchelchen lagen hintereinander.

Am wenigsten entfernten sich bei Doppeldaumen die am gewöhnlichen Daumen in der Zweizahl ganz konstanten Sesambeine der Articulatio metacarpophalangea I von der Norm.

Nur einmal wurden sie auch hier ganz vermißt. Dreimal war nur eins vorhanden und zwar das ulnare, dem gewöhnlich stärkeren ulnaren Daumen entsprechende. Neunmal dagegen verhielten sie sich der Zahl nach wie in der Norm.

Wenn man die aus dieser Zusammenstellung sich ergebende Tatsache würdigt, daß nämlich bei dem unpaaren Sesamoid der kleinen Zehe am liäufigsten eine Verdoppelung bestand, während bei Daumenverdoppelung bei weitem am häufigsten die normale Zweizahl zur Beobachtung kam, so steht sie mit der Spaltungstheorie, nach welcher die Hyperdaktylie durch eine Einwirkung von außen auf die ursprünglich einfache, indifferente Anlage des Endgliedes hervorgerufen wird, durchans im Einklange. Die wenigen Ausnahmen dürften bei der großen Variabilität, welche die anatomischen Verhältnisse der menschlichen Hyperdaktylie darbieten, nicht wunder nehmen. Dabei ist aber die Einschränkung zu machen, daß in zwei Fällen von Doppeldaumen, bei welchen eine Verdoppelung resp. Spaltung des Metacarpus I bestand, die beiden Ossicula nur dem stärkeren ulnaren Daumen angehörten, während der schwächere radiale Daumen keine besaß. Dasselbe scheint anch in einem Falle bei einfachem Metacarpus I vorgelegen zu haben. Auch können in den obigen vier Fällen von verdoppelter kleiner Zehe die nach Thilenius hier ursprünglich paarig angelegten Sesamoide in der Zweizahl persistent geblieben sein.

2.

\title{
Bemerkungen zum Anhang der Fischlerschen Arbeit: Über experimentell erzeugte Fettsynthese am überlebenden Organ usw.
}

Von

Dr. med. F. Hagemeister,

Assistenzarzt an Dr. Borcks Privatklinik in Rostock.

Im 174. Band dieses Archivs berichtet Fis chler über "experimentell erzeugte Fettsynthese am überlebenden Organ" and liefert den wertvollen Nachweis, daB Fett in der von der normalen Zirkulation getrennten Niere ans der die Fettkomponenten enthaltenden Flüssigkeit, mit der sie künst- 
lich durchströmt wird, entstehén kann. In einem Anhange zu dieser Arbeit wendet $\mathrm{Fischler}$ sich gegen die von uns vertretene Anschaung, ${ }^{1}$ ) daß arterielle Hyperämie immer zu Fettschwund führt, and meint, es gäbe, allerdings seltene, Fälle, in denen man bei arterieller Hyperaemie Fettablagerung beobachten könne. Er beruft sich dabei, als auf das reinste ihm gerad'e gegenwärtige Beispiel, auf das Resultat einer früheren Untersuchung, ${ }^{2}$ ) bei der er festgestellt hat, daß beim Kaninchen nach Exstirpation einer Niere die andere eine große Menge feinster Fettröpfchen' in den Epithelien führte. Da es eine durch zahlreiche Untersuchungen bestätigte Tatsache ist, daB nach Exstirpation der einen Niere in der zurückbleibenden Hyperämie (und als Folge davon gesteigerte Funktion und Hyperplasie) auftritt, so darf nach unserer Auffassung in der im Körper verbleibenden Niere kein Fett zu finden sein, und jener Befund Fischlers wäre demnach geeignet, die Richtigkeit unserer These in Frage zu stellen.

Von den Versuchen, die F ischler in seiner früheren Abhandlung anführt, gehen uns hier nur die vier an, in denen er zunächst die eine Niere und später, in allmählich zunehmenden Zwischenräumen, die andere exstirpiert hat. Dabei ergab sich, daß von den im Körper gebliebenen Nieren diejenige, die am frühesten exstirpiert war (nach $7 \frac{1}{2}$ Stunden) den höchsten Fettgehalt aufwies, während die am spätesten exstirpierte (nach 3 Wochen) am wenigsten fethaltig war. Das 5. Kaninchen seiner Versuche muB deswegen aus unserer Betrachtung ausscheiden, weil dasselbe infolge einer Verblutung zugrunde ging; es ist bekannt, daß Organe von Tieren, die an langsamer Verblutung gestorben sind, regelmäBig Fett enthalten, wie wir im Sinne unserer eigenen Arbeit annehmen, deswegen, weil der Blutdruck und damit auch die Bewegung der Gewebsflüssigkeit herabgesetzt sind, so dab sich in den Zellen die Fettsynthese vollziehen muB.

Da, je später die zurückgebliebene Niere exstirpiert war, desto weniger Fett angetroffen wurde, so lag die Vermutung nahe, dab die von F ischler angewandte Narkose mittels Chloralhydrats und Chloroforms in einem Zusammenhang mit dem Auftreten des Fettes stehe.

Um dieser Frage näher zu treten, machten wir eine Reihe von 10 Tierversuchen, und zwar in der Weise, daß wir ohne Anwendung irgend eines Narcoticums den Kaninchen zuerst die linke Niere exstirpierten und dann nach verschieden langen $Z$ wischenrärumen die rechte. Wir haben

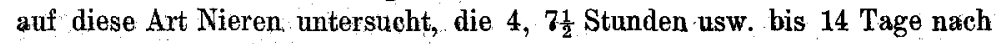
Entfernung der linken Niere herausgenommen waren. Die Nieren wurden mit Fixierung in Formol, Färbung des Fettes mit Osmium und mit Sudanlösung behandelt.

1) F. Hagemeister, Beiträge zur Kenntnis des Fettschwundes und. der Fettbildung in ihrer Abhängigkeit von Zirkulationsänderungen. Dieses Archiv, 172. Band, 1903.

2) F. Fischler, Ober den Fettgehalt von Niereninfarkten, zugleich ein Beitrag aur Frage der. Fettdegeneration. Dieses Archiv, 170. Band, 1902. 
Bei der Untersuchung stellte sich heraus, daß, abgesehen von einigen Fetttröpfélen in ganz vereinzelten Präparaten, die Schnitte keine Spur von Fett aufwiesea. Die wenigen Fetttröpfehen, die wir an einzelnen Stellen sahen, in den Sudanpräparaten etwas zahlreicher tond dicker als in den Osmiumpräparaten, fanden sich in der Grenzzone zwischen Rinde und Mark, also einem Gebiet, wo physiologischerweise in derselben oder in größerer Menge Fett beobachtet wird.

Aus diesen Untersuehangen geht hervor, dả in der nach Exstirpation der anderen hyperämischen Niere keine Fettvermehrang auftritt, sofern der Versuch rein, d. h. ohne andere Eingriffe, denen unter Umständen Fettvermehrung in den Organen zuzuschreiben ist, angestellt wird.

Wir wären nun noch einen Schritt weiter gegangen und hätten Parallelversuche unter Narkose durch Chloralhydrat und Chloroform gemacht, doch war vorauszusehen, daß, wenn überhaupt, bei der so außerordentlich individuell schwankenden Wirkung der: Nareatica nur sehr zahlreiche und mannnigfach variierte Versuche ein befriedigendes Ergebnis geliefert hätten; äußerer Umstände halber mußten wir darauf verzichten. Auch ist es durch sehr zahlreiche Untersuchnngen zur Genüge bekannt, daß Chloralhydrat bei seiner Wirkung auf das Zentralnervensystem sehr häufig den Blutdruck herabsetzt, indem der Gefäßtonus vermindert wird. Nun ist mit dieser Blutdruckherabsetzung verbunden eine Blutstromverlangsamung, die ihrerseits wieder zu einer Verlangsamung des Lymphstroms führt. Gerade dies sind aber die Faktoren, die, wie wir in unserer Arbeit nachgewiesen haben, den Fettaufbau herbeiführen.

Es geht nicht an, den hierin enthaltenen Einwand gegen die ZweckmäBigkeit der Chloralhydratnarkose bei das Fett betreffenden Untersuchungen, wie Fischler tut, mit der Behauptung entkräften zu wollen, daß nur bei chronischem Chloralgebrauch Verfettung auftrete; die Tatsachen, daß einerseits bei der Wirkung der Narcotica auf den Blutdruck die Art und Weise der Dosierung sehr zurücktreten, und andererseits die Fettsynthese sich bekanntermaßen sehr schnell vollziehen kann, verbieten dies.

Fischler sucht freilich das Auftreten des Fettes ,nach großen Chloralgaben" gerade gegen unsere Theorie der Abhängigkeit des Fettes von dem Verhalten der Zirkulation zu verwerten, indem er das Chloral „eine allgemeine Hyperämie" hervorrufen läßt. Er vergißt hinzuzufügen, daß diese "allgemeine Hyperämie" nicht mit Strombeschleunigung, sondern mit Stromverlangsamung verbunden ist, jener Befund somit nicht gegen, sondern für unsere Theorie spricht. Daß dabei "Degenerationserscheinungen", worunter Fischler wohl morphologische Abweichungen neben dem Auftreten des Fettes versteht, fehlen könnten, ist belanglos.

Die weiteren Einwände Fischlers gründen sich auf seine Untersuchungen über das Fett des anämischen Niereninfarktes. Da dieser Gegenstand in einer jüngst erschienenen Arbeit ${ }^{1}$ ) aus dem Rostocker

1) Brodersen, Die Veränderungen in der Niere nach zweistündiger Unterbindung der Arteria renalis als Folgen einer veränderten Durchströmung des Gefäßes. Dissertation, Rostock, 1904. 
Institut noch eimmal behandelt worden ist, dürfen wir auf diese, sowie anf die das Fett in der Leber in seiner Abhängigkeit von der Zirkulation behandelnden Untersuchungen von Tischner ${ }^{1}$ ) verweisen; in der gleichen Absicht der Kürze verzichten wir darauf, die theoretischen Bedenken Fischlers zu erörtern.

1) Tischner, Vergleichende Untersuchungen zur Pathologie der Leber. Dieses Archiv, 175. Band, 1904. 